\title{
Analysis of Time Trend of Cost of Medications
}

\author{
Rajan Bhagat ${ }^{1}$, Nusrat Shafiq ${ }^{2}$, Samir Malhotra ${ }^{3}$
}

\begin{abstract}
Background: Increase in prices of drugs has always been a topic of concern across the world. In countries like India, where people have to bear this expenditure out of pocket, rise in prices of drugs is a major issue.

Objective: To find out the pattern of changes in prices of some commonly used drugs over a period of 15 years.

Materials and methods: We selected some commonly used drugs used in the treatment of cardiovascular system (CVS), central nervous system (CNS), respiratory, hematological, gastrointestinal, and endocrine diseases. We also included some other drugs like antimicrobials, anti-allergics, and nonsteroidal anti-inflammatory drugs (NSAIDs). Information on the prices of drugs was extracted at four time points: years 2001, 2006, 2011 , and 2016. Percentage change in prices over a period of 15 years was calculated. We followed the time course of price variation of the same dosing strengths of the highest and lowest priced brands, out of the various brands available. We categorized the drugs into three categories: more than or equal to $100 \%$ increase in price, "decrease" and "no change."

Results: Out of total 117 drugs, 28 drugs (24\%) showed an increase of more than $100 \%$ over this period. Among lowest priced brands, there was a mean [standard deviation (SD)] increase of 222\% (142), and among highest priced brands, there was an average increase of 244\% (205). Fifty-nine (50\%) drugs showed "no change" or "decrease" in price, the reason for which in 31 (52.5\%) came under price control.

Conclusion: Since the prices of raw materials tend to decline over time, the hike in drug prices appears unjustified.

Keywords: Commonly used drugs, Drug price, Increase over time.

Journal of Postgraduate Medicine, Education and Research (2020): 10.5005/jp-journals-10028-1357
\end{abstract}

\section{INTRODUCTION}

Rise in the price of drugs has always been a topic of concern all over the world. In the year 2015, Turing Pharmaceuticals increased the price of Daraprim (pyrimethamine) in the USA by 5,000\%, from $\$ 13.50$ per dose to $\$ 750 .{ }^{1}$ Another recent example is of EpiPen (epinephrine), the price of which was increased from $\$ 100$ (year 2009) to $\$ 600$ (year 2016) by Mylan. ${ }^{2}$ In countries like India, where patients pay for medicines out of their pockets, ${ }^{3}$ this is a major issue. Number of Indians falling below the poverty line because of healthcare expenditure may increase to almost $7 \%$ of the country's population. ${ }^{4}$ There is anecdotal evidence that prices of medicines have increased in India over past few years, but no study has systematically evaluated the same.

To keep medicines affordable for majority of population, the National Pharmaceutical Pricing Authority (NPPA) was enacted in 1997. ${ }^{5}$ In addition, some drugs from National Essential Medicines List (NLEM) were put under price control, for example, ramipril, carbamazepine, levofloxacin, and ranitidine. ${ }^{6}$

Due to the opposing factors of government control and a tendency by the companies to increase prices, it remains unclear how the prices of medicines have varied over the past decade or so. Therefore, in this study, we planned to systematically evaluate fluctuations in prices of commonly used drugs with specific aim of establishing if there have been excessive price increases.

\section{Materials and Methods}

\section{Study Design}

A planned review of resources for the structured data extraction and analysis.

To evaluate changes in prices occurring over time, we included commonly used drugs in the treatment of cardiovascular system (CVS), central nervous system (CNS), respiratory, hematological, endocrine systems, dyslipidemia, and gastrointestinal disorders.
${ }^{1}$ Parexel International, Mohali, Punjab, India; Department of Pharmacology, Postgraduate Institute of Medical Education and Research, Chandigarh, India

${ }^{2,3}$ Department of Pharmacology, Postgraduate Institute of Medical Education and Research, Chandigarh, India

Corresponding Author: Nusrat Shafiq, Department of Pharmacology, Postgraduate Institute of Medical Education and Research, Chandigarh, India, Phone: +91 9478000822, e-mail: nusrat.shafiq.pgi@gmail.com

How to cite this article: Bhagat R, Shafiq N, Malhotra S. Analysis of Time Trend of Cost of Medications. J Postgrad Med Edu Res 2020;54(2): 34-36.

Source of support: Nil

Conflict of interest: None

In addition, we also included antimicrobials like amoxicillin and azithromycin, nonsteroidal anti-inflammatory drugs (NSAIDs) like diclofenac sodium, anti-allergics like cetirizine, and some vitamin and mineral preparations. Within each category, we selected drugs based on volume of sales. For example, in lipid-lowering category, we included atorvastatin; in CVS, metoprolol and ramipril; in CNS, diazepam and lorazepam; in respiratory system, salbutamol; in hematological system, heparin; in endocrine system, metformin; and in gastrointestinal system, ranitidine and omeprazole. In our study, we included drugs used systemically and did not evaluate local/topical formulations. Ethical approval was not necessary because this study did not involve any human participation.

We decided to review the prices of the selected drugs over a period of 15 years at intervals 2001, 2006, 2011, and 2016. Information about prices was retrieved from one or more of the following sources: Indian Drug Review, Current Index of Medical Specialties, or Drugs Update.

We started by extracting the following information for each of the selected drug: For the year 2001, brand being sold at the highest 
price and the lowest price. We followed the time course of price variation of the same dosing strengths of the highest and lowest priced brands, out of the various brands available, at the following three times: 2006, 2011, and 2016. In case there was a change in the brand with the highest or the lowest price, we used, for our comparison, brands with highest or lowest prices. For example, if brand " $X$ " was the lowest priced brand in 2001, but it did not appear in the 2016 list, and brand " $Y$ " was the lowest priced brand in 2016 list, we used brand " $Y$ " for comparison.

After retrieving prices of the highest and the lowest priced brands of our selected drugs during all the four periods (2001, 2006, 2011, and 2016), we then calculated the percentage changes in prices across the last 15 years using the formula:

$$
\frac{\text { Next }- \text { Previous }}{\text { Previous }} \times 100
$$

We then categorized the selected drugs into three categories: drugs with equal to or more than $100 \%$ increase, drugs with either "no change" or "decrease" in prices. For the drugs, which were categorized as "no change" or "decrease," we looked at the NLEM to see how many of these drugs were in the list. We also calculated the average percentage change for all the drugs and for drugs with more than $100 \%$ increase in their prices.

The trend of price change during this period of 15 years was plotted, which showed an increase of $100 \%$ or more over the evaluation period. We plotted the trend of change in prices using MS Excel.

\section{Results}

We evaluated price changes of 117 drugs across various therapeutic categories (Table 1).

In total, 28 drugs (24\%) showed an increase of more than $100 \%$ over this period. When we evaluated the lowest priced brands, there was a mean (SD) increase of $222 \%$ (142) for the drugs (Table 2).

When we evaluated the highest priced brands, there was an average increase of $244 \%$ (205).

There was no clear-cut trend in the pattern of increase in prices, and prices of some drugs increased gradually although some showed an abrupt rise at one particular time point (Figs 1 and 2).

Fifty-nine (50\%) drugs showed "no change" or "decrease" in price over this period. Out of these drugs, 31 (52.5\%) come under price control after inclusion of their names in NLEM.

Table 1: Number of drugs in various therapeutic categories and their respective percentages out of total number of drugs (117)

\begin{tabular}{llll}
\hline S.no. & Therapeutic category & $\begin{array}{l}\text { Number of } \\
\text { drugs }\end{array}$ & $\begin{array}{l}\text { Percentage (out } \\
\text { of total) }\end{array}$ \\
\hline 1. & Antimicrobials & 34 & 28.8 \\
2. & Central nervous system & 27 & 22.8 \\
3. & Cardiovascular system & 13 & 11 \\
4. & Gastrointestinal system & 10 & 8.5 \\
5. & Endocrine system & 9 & 7.6 \\
6. & Hematological system & 7 & 6 \\
7. & Respiratory system & 6 & 6 \\
8. & Anti-allergics & 4 & 3.4 \\
9. & Lipid-lowering agents & 1 & 0.8 \\
10. & Multivitamin and minerals & 1 & 0.8 \\
\hline
\end{tabular}

\section{Discussion}

We observed how the prices of some commonly used drugs have changed across the last 15 years, i.e., from year 2001 to year 2016. In total, 28 drugs (24\%) showed an increase of more than $100 \%$ during this period. Lorazepam, metoprolol, and chlordiazepoxide being the leading candidates with more than $500 \%$ rise in their prices. These were followed by isosorbide-5-mononitrate, clonazepam, phenytoin, indomethacin, fluconazole, diazepam, diclofenac

Table 2: Percentage changes in prices of drugs that showed more than $100 \%$ increase in the lowest priced brand list. Average increase in prices being the $222 \%$

\begin{tabular}{ll}
\hline Drug (lowest priced brand) & $\begin{array}{l}\text { Percentage change in } \\
\text { price over 15 years }\end{array}$ \\
\hline 1. Metoprolol & 616.8 \\
2. Chlordiazepoxide & 504.8 \\
3. Isosorbide 5-mononitrate & 450 \\
4. Clonazepam & 372.2 \\
5. Phenytoin & 338.79 \\
6. Indomethacin & 275.42 \\
7. Fluconazole & 236.84 \\
8. Digoxin & 200.51 \\
9. Paracetamol & 190.4 \\
10. Grilinctus cough syrup & 178.46 \\
11. Clopidogrel & 162.94 \\
12. Famotidine & 154.58 \\
13. Doxycycline & 136.36 \\
14. Imipramine & 128.8 \\
15. Metoclopramide & 128.3 \\
16. Pantoprazole & 128.2 \\
17. Fluoxetine & 115.8 \\
18. Clozapine & 109.9 \\
19. Corex cough syrup & 109.1 \\
20. Cetirizine & 108.3 \\
21. Sodium valproate & 102.6 \\
Average increase & 222 \\
\hline
\end{tabular}

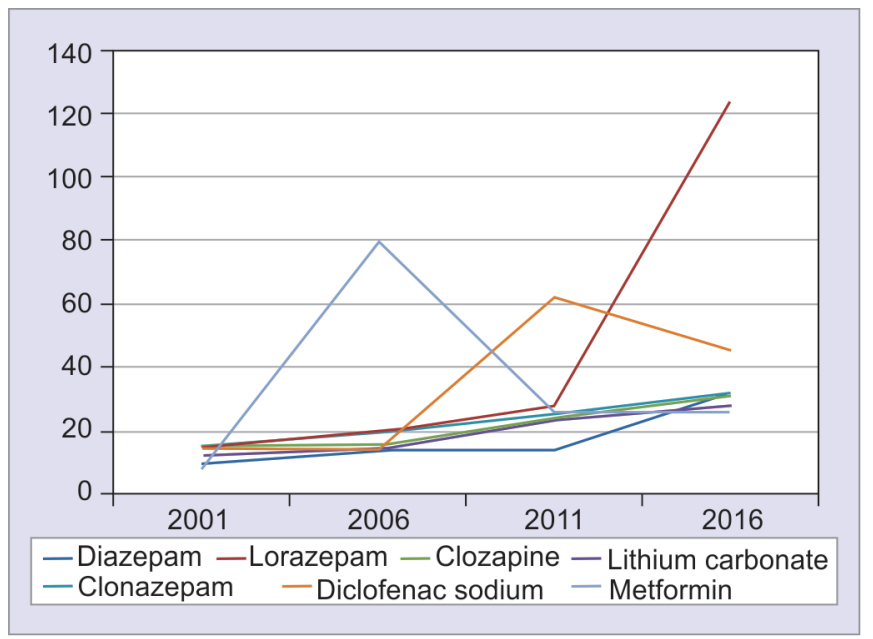

Fig. 1: Percentage change: diazepam-215.39, lorazepam-735.2, clozapine-103.27, lithium carbonate-129.5, clonazepam-113.3, metformin -198.8 , and diclofenac sodium -213.79 


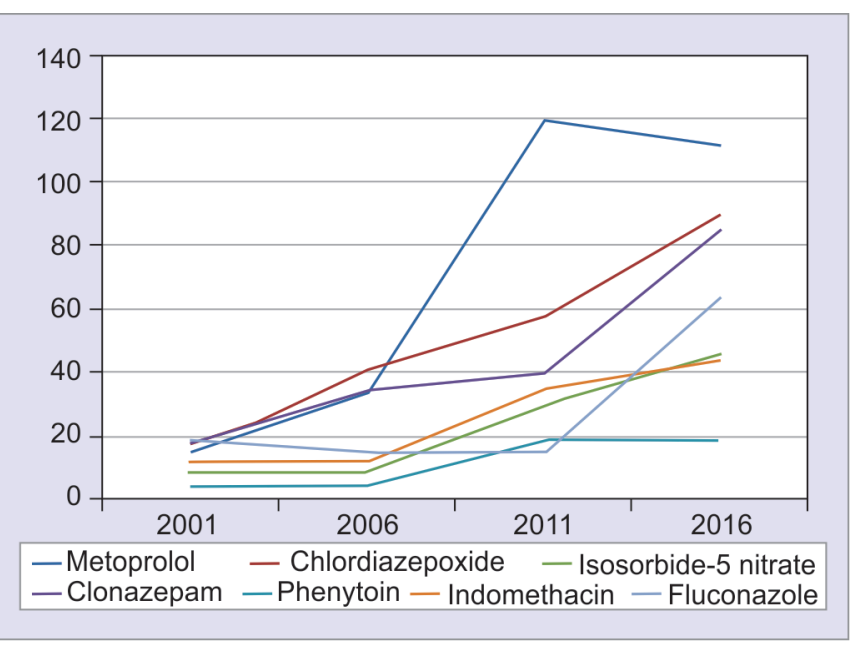

Fig. 2: Percentage change: metoprolol -616.8 , chlordiazepoxide-504.8, isosorbide-5-mononitrate-450, clonazepam-372.2, phenytoin-338.8, indomethacin -275.4 , and fluconazole -236.8

sodium, and digoxin in which percentage change in prices was an increase ranging between 200 and 500\%. All these drugs are available as generic drugs in India. Increase in prices of generic drugs has been an area of concern even in developed countries. ${ }^{7}$ An old drug like digoxin was seen to be the most expensive drug in a recent study. ${ }^{8}$

The inflation rates in India over this period have varied between 3 and $15 \%{ }^{9}$ and although it would be tempting to calculate inflationadjusted changes in the prices of drugs, this is not the case in case of medicines. The prices of drugs are known to decline over time due to entry of competing drugs in the market as well as the fact the prices of the bulk drugs fall with time. ${ }^{10}$

Healthcare expenditure has a major impact on financial condition of the vast majority of population in India as most of this expenditure is out of pockets. ${ }^{4}$ Among the various healthcare costs, drugs constitute a large chunk of health-related expenditure. ${ }^{11}$ The government has various ways to keep the prices of medicines in check. One of them is the NPPA. For example, recently, prices of stents have been decreased by the government. ${ }^{12}$ Also, prices of some of the drugs like methyldopa, griseofulvin, and amphotericin B have been fixed. ${ }^{5}$ Another mechanism is through the NLEM-drugs included in NLEM are put under price control, for example, carbamazepine, levofloxacin, and rantidine. ${ }^{6}$

It is indeed a surprising finding as to how the companies have managed to increase the prices of some of the most commonly used drugs in spite of the presence of the above-mentioned regulations. This calls for brainstorming among the various stakeholders to find out ways of how to keep the medicines affordable.

This new adverse drug reaction, type $F$ (financial), must be considered as important as the other established types ( $A, B$, etc.) and deserves similar attention.

\section{References}

1. Drug Goes From $\$ 13.50$ a Tablet to $\$ 750$, Overnight. www.nytimes. com/.../a-huge-overnight-increase-in-a-drugs-price-raises-protests. Accessed Sep 20, 2016.

2. 5 things we learned from EpiPen price hike hearing. www. usatoday.com/story/money/2016/09/22/epipen-congresshearing.../90827270/. Accessed Sep 22, 2016.

3. Kotwani A, Ewen M, Dey D, et al. Medicine prices and availability at six sites in India: using WHO-HAI medicine prices methodology. Indian J Med Res 2007;125(5):645-654.

4. Berman $P$, Ahuja R, Bhandari L. The impoverishing effect of healthcare payments in India: new methodology and findings. Econ Polit Wkly 2010;45:65-71.

5. National Pharmaceutical Pricing Authority: Whats New. www. nppaindia.nic.in/frequent.html. Accessed January 17, 2017.

6. Compendium of Prices - NPPA. www.nppaindia.nic.in/ compendium2016. Accessed January 15, 2017.

7. Alpern JD, Stauffer WM, Kesselheim AS. High-cost generic drugs: implications for patients and policymakers. N Engl J Med 2014;371(20):1859-1862. DOI: 10.1056/NEJMp1408376.

8. Hauptman PJ, Goff ZD, Vidic A, et al. Variability in Retail Pricing of Generic Drugs for Heart Failure. JAMA 2016.

9. Historic inflation India - historic CPI inflation India - Inflation.eu. www. inflation.eu/inflation-rates/india/historic-inflation/cpi-inflationindia.aspx. Accessed January 20, 2017.

10. Exploratory Study on Active Pharmaceutical Ingredient Manufacturing for Essential Medicines. apps.who.int > All > Quality and Safety: Medicines > Quality Assurance. Accessed January 20, 2017.

11. National Health Expenditures 2015 Highlights. https://www.cms.gov/ research-statistics.../nationalhealthexpenddata/.../highlights.pdf. Accessed January 28, 2017.

12. National Pharmaceutical Pricing Authority slashes stent prices by up to $85 \%$. timesofindia.indiatimes.com > India News. Accessed February 20, 2017. 